\title{
TENDENCIA ESPACIAL Y TEMPORAL DE EVENTOS CLIMÁTICOS EXTREMOS EN EL VALLE GEOGRÁFICO DEL RÍO CAUCA
}

\section{SPATIAL AND TEMPORAL TRENDS OF EXTREME CLIMATE EVENTS IN GEOGRAPHICAL VALLEY OF CAUCA RIVER}

\author{
Daniel Elías Cuartas ${ }^{1}$, Diana María Caicedo ${ }^{2}$, Delia Ortega ${ }^{3}$, Faisury Cardona ${ }^{4}$, \\ Yesid Carvajal $^{5}$, Fabián Méndez ${ }^{6}$
}

\begin{abstract}
${ }^{1}$ Geógrafo. Universidad del Valle, Calle 4B No. 36-140, Edificio 118, espacio 215, Cali - Valle, Colombia, e-mail: daniel.cuartas@correounivalle.edu.co; ${ }^{2}$ Médico, Magister en epidemiología. Universidad del Valle, Calle 4B No. 36-140, Edificio 118, espacio 215, Cali - Valle, Colombia, e-mail: dmcaicedo@grupogesp.org; ${ }^{3}$ Estadístico, Magister en epidemiología. Universidad del Valle, Calle 4B No. 36-140, Edificio 118, espacio 215. Universidad Santiago de Cali, Calle 5 No. 62-00, Cali - Valle, Colombia, e-mail: dortega@grupogesp.org; ${ }^{4}$ Ingeniera Agrícola. Universidad del Valle, Calle 13 No. 100-00, Edificio 344, espacio 2007, Cali - Valle, Colombia, e-mail: faisury.cardona@correounivalle.edu.co; ${ }^{5}$ Ingeniero Agrícola, Maestría en suelos y aguas, Maestría en hidrología aplicada, Ph.D. en hidrología y medio. Universidad del Valle, Calle 13 No. 100-00, Edificio 344, espacio 2007, Cali - Valle, Colombia, e-mail: yesid.carvajal@correounivalle.edu.co; ${ }^{6}$ Médico, Magister en epidemiología, Ph.D. Public Health. Universidad del Valle, Calle 4B No. 36-140, Edificio 118, espacio 215, Cali - Valle, Colombia, e-mail: fmendez@grupogesp.org
\end{abstract}

Rev. U.D.C.A Act. \& Div. Cient. 20(2): 267-278, Julio-Diciembre, 2017

\section{RESUMEN}

Los eventos climáticos extremos pueden incrementar la ocurrencia del dengue y de enfermedades diarreicas agudas, en áreas altamente sensibles, con poco nivel de adaptación a condiciones cambiantes, bajo el escenario del cambio climático. El objetivo de este estudio fue describir la tendencia espacial y temporal de los eventos extremos de temperatura y de precipitación en el valle geográfico del río Cauca. Se controló la calidad y la homogenización de los datos climáticos. Se desarrolló un análisis robusto no paramétrico de tendencia de eventos climáticos extremos para precipitación y temperatura. Se analizaron 24 estaciones climatológicas en el Valle geográfico del río Cauca. El análisis regional para precipitación y temperatura mostró una tendencia al incremento de los índices de eventos extremos y el análisis local identificó una distribución no homogénea, en el área de estudio. Los resultados se discutieron en virtud de los posibles impactos en salud, específicamente, para dengue y enfermedad diarreica aguda. El aumento de los días lluviosos y de la cantidad de precipitación podrían generar un aumento en la densidad vectorial y la longevidad larval de Aedes aegypti, además causar el desbordamiento de ríos y canales, con combinación de aguas servidas y resuspensión de sólidos, produciendo el aumento de microorganismos patógenos en el agua de consumo. Adicionalmente, la tendencia al incremento de la temperatura podría acortar el ciclo de vida del Aedes y la replicación interna del virus con mayor probabilidad de transmisión del dengue, al mismo tiempo, que aumentaría la sobrevida de bacterias y de protozoos en aguas negras, superficiales y suelo, aumentando la ocurrencia de las EDA.

Palabras clave: Efectos del clima, temperatura, lluvia, descriptores en ciencias de la salud.

\section{SUMMARY}

Extreme climate events can impact the occurrence of dengue and diarrheal diseases. The objective of this paper was to describe the spatial and temporal trend of extreme temperature and precipitation events in the geographical Valley of Cauca River. Quality and homogenization of climate data was monitored. Robust nonparametric trend analysis of extreme weather events for precipitation and temperature was developed. 24 weather stations were analyzed in the geographical Valle del Cauca River. The regional trend analysis showed a trend of increasing rates of extreme events for precipitation and temperature. Meanwhile, local analysis identified that this trend is not homogeneous in the study area. The rise in volume of precipitation and in the number of rainy days can increase Aedes aegypti, density and larval longevity and also cause overflowing of rivers and canals, with combination of sewage and resuspension of solids and pathogenic microorganisms in water. In addition, the rise in 
temperature shortens Aedes life cycle and viral replication in mosquitoes with higher probability of dengue transmission and meanwhile increases the survival of bacteria and protozoa in surface sewage and soil causing more occurrence of EDA.

Key words: Climate effects, temperature, rain, dengue, diarrhea.

\section{INTRODUCCIÓN}

El cambio climático (CC) es una amenaza para la salud humana y afecta, de manera diferencial, a países de bajos ingresos y a poblaciones vulnerables. Los cambios en la temperatura, la precipitación y la ocurrencia de eventos extremos impactan en diferentes eventos en la salud humana, incluyendo un aumento en la carga de morbilidad y de mortalidad por enfermedades infecciosas (Patz et al. 2008).

En la actualidad, se presentan cambios en la tendencia de ocurrencia de eventos climáticos extremos, caracterizados por el incremento en la frecuencia e intensidad de los mismos. El análisis de estos eventos, se encuentra limitado por la calidad de datos disponibles y por las escalas de medición. Adicionalmente, dado que son eventos no esperados tienen el potencial de impactar negativamente la sociedad, por lo que se requiere conocer su ocurrencia y tendencia para evaluar la vulnerabilidad a escala local y desarrollar estrategias de adaptación (Intergovernmental Panel on Climate Change - IPCC, 2012).

En algunas regiones, sobre todo en países ecuatoriales, como Colombia, se están presentando cambios; en Cundinamarca, se identificó una tendencia creciente en índices de eventos extremos de temperatura y de precipitación a escala trimestral (Montealegre, 2012). Los efectos se sentirán en diferentes dimensiones, como agricultura, ecosistemas, abastecimiento de agua potable, infraestructura y salud, entre otros. En la tercera comunicación nacional de Colombia a la convención marco de las Naciones Unidas sobre cambio climático, la salud es una de las seis dimensiones empleadas para el análisis de vulnerabilidad y riesgo e identificaron que la salud, a nivel nacional, tiene una amenaza alta (IDEAM et al. 2017).

Los nexos entre el clima y los mecanismos de transmisión de las enfermedades infecciosas son múltiples, están especialmente documentados para enfermedades transmitidas por vectores (ETV) (Brunkard et al. 2008); no obstante, existen brechas de conocimiento sobre cómo los cambios globales y locales en el clima podrán afectar la incidencia, en las enfermedades infecciosas relacionadas con el agua, como ETV y enfermedades diarreicas agudas (EDA) (Kovats et al. 2005; McMichael \& Woodruff, 2005).
Al ser eventos en salud complejos en los que intervienen diferentes dimensiones (políticas, económicas, sociales, comportamentales, entre otras), también podrían abarcar cambios en el comportamiento de las personas que aumenten la exposición a la infección (Forero et al. 2014); por ejemplo, precipitaciones extremas pueden producir cortes en el suministro de agua, lo que lleva a las personas a almacenar agua y aumentar la posibilidad de existencia de criaderos de Aedes aegypti o la contaminación por microrganismos en el agua de consumo. Cai et al. (2015) estiman que en los próximos años ocurrirán eventos extremos más intensos y complejos asociados, tanto a La Niña como a El Niño, situación que se convierte en un reto para diversos sectores, entre ellos, la salud.

Para el dengue, una enfermedad causada por un arbovirus y transmitida por mosquitos del género Aedes (especies Ae. aegypti y Ae. albopictus) (Velandia \& Castellanos, 2011), la temperatura influencia la tasa de reproducción vectorial y la replicación viral dentro de los mosquitos. Adicionalmente, la precipitación influye en la disponibilidad de criaderos para los mosquitos y en la distribución geográfica (Morin et al. 2013). Investigaciones en países tropicales y subtropicales han encontrado asociaciones positivas significativas de los casos de dengue con las variables climáticas y con el fenómeno El Niño Oscilación del Sur - ENOS (Colón-González et al. 2013; Chen et al. 2012; Naish et al. 2014). Adicionalmente, Morin et al. (2013) concluyen que dichos resultados dependen de los contextos locales.

Por su parte, para las EDA, aumentos de la precipitación influencia la cantidad de microorganismos patógenos en aguas superficiales y subterráneas, mientras que su disminución puede llevar a una mayor concentración de los patógenos en el agua. Además, incrementos de la temperatura influye en la sobrevida de los microorganismos bacterianos y una menor humedad relativa se ha relacionado con una mayor sobrevida de patógenos virales (Bandyopadhyay et al. 2012; Chen et al. 2012; Moors et al. 2013); lo anterior se presenta, tanto en temporadas lluviosas como secas (Friedrich, 2013).

En este sentido, bajo un proyecto marco que evaluó la vulnerabilidad en salud al cambio climático, el objetivo de este estudio fue describir la tendencia espacial y temporal de eventos extremos de temperatura y de precipitación en el valle geográfico del río Cauca (VGRC) y los resultados se discutieron en términos de los posibles impactos en la ocurrencia de dengue y diarrea.

\section{MATERIALES Y MÉTODOS}

Área de Estudio. El VGRC está ubicado en el suroccidente colombiano y discurre entre las cordilleras Occidental y 
Central; tiene un área aproximada de $18.000 \mathrm{~km}^{2}$, hasta La Virginia, en Risaralda. Presenta elevaciones que varían entre $900 \mathrm{msnm}$ y $4.000 \mathrm{msnm}$. El clima de la región, se rige por diferentes factores que controlan el clima en Colombia y presenta dos temporadas de aumento de lluvias (marzo-mayo y septiembre-noviembre) y dos temporadas donde la lluvia disminuye (diciembre-enero y junio-agosto) (Poveda et al. 2011). Estos factores generan una alta variabilidad espacial y temporal del clima en el VGRC y una gran vulnerabilidad a eventos climáticos extremos (Hoyos et al. 2012; Reboita et al. 2012).

Control de calidad y homogenización de datos climáticos. Los registros históricos diarios de precipitación y de temperatura máxima y mínima, se recopilaron de las bases de datos de entidades públicas y privadas. Se realizó una preselección de estaciones que contenían más del $80 \%$ de los datos en sus registros históricos, en diferentes periodos de tiempo (1 estación con datos de temperatura, entre 01/01/1973 31/12/2013; 1 estación con datos de precipitación, entre 01/01/1965 - 31/12/2009 y 24 estaciones con datos de temperatura y precipitación, entre 01/01/1998 y 31/12/2013).

Se aplicaron filtros para detectar valores erróneos, como precipitaciones o temperaturas negativas. Las pruebas de tolerancia, se hicieron para valores que resultaron atípicos para 3,5 desviaciones estándar (National Climatic Data Center - NCDC, 1997) y los valores atípicos de cada estación, se compararon con las estaciones vecinas. El análisis de consistencia interna inspeccionó la coherencia entre elementos asociados, como el caso de la temperatura máxima, que no puede ser inferior a la temperatura mínima. La coherencia temporal consistió en la observación de la consistencia de los datos con cambios esperados, como la ocurrencia del fenómeno ENOS. Finalmente, la coherencia espacial permitió comparar los valores atípicos con estaciones próximas (Aguilar et al. 2003). En consecuencia, 25 estaciones fueron descartadas, por tener más del $20 \%$ de datos perdidos.

Se realizaron pruebas de homogeneidad en las 24 estaciones y 8 requirieron homogenización. Este procedimiento, se desarrolló con Rclimdex y se empleó una técnica indirecta, por ser datos diarios, que no requirió la creación de una serie de referencia, la detección de puntos de corte y ajustar los datos (Peterson et al. 1998), por medio de los módulos RHtest_dlyPrcp y Rclimtool.

Selección de índices de eventos extremos. La selección de índices de eventos climáticos extremos, se realizó a partir de los 27 indicadores descriptivos, propuestos por el Equipo en Detección de Cambio Climático e Índices (ETCCDI, por sus siglas en idioma inglés) y otros 27 índices propuestos para Latinoamérica, por parte por sus siglas en idioma inglés). Los indicadores hacen referencia a la frecuencia, la amplitud y la persistencia (M.G. et al. 2009) de los eventos climáticos y están clasificados en 8 categorías: nubosidad, frío, sequía, calor, humedad, lluvia, temperatura y compuestos.

Se realizó una revisión de los índices que pudieran estar relacionados con las condiciones ambientales, que favorecen la ocurrencia de dengue y EDA. Se seleccionaron tres tipos de índices, según su ocurrencia en el tiempo, conteo de días, agregación en días consecutivos y agregación mensual y se calcularon, de acuerdo a la definición en escala mensual TX> TX90P (Tabla 1).

Tabla 1. Indicadores de eventos extremos seleccionados.

\begin{tabular}{|c|l|c|c|}
\hline Nombre & \multicolumn{1}{|c|}{ Definición } & Unidades & \multicolumn{1}{|c|}{ Indica } \\
\hline CWD & Número máximo mensual de días húmedos consecutivos RR $>=1 \mathrm{~mm}$ & Días & Frecuencia \\
\hline TWD & Total mensual de días húmedos RR $>1 \mathrm{~mm}$ & Días & Frecuencia \\
\hline PRCPTOT & Precipitación mensual total en días húmedos & mm & Magnitud \\
\hline RX5day & Precipitación máxima mensual en 5 días consecutivos & mm & Magnitud \\
\hline TDD & Total mensual de días secos RR $<1 \mathrm{~mm}$ & Días & Frecuencia \\
\hline CDD & Número máximo de días secos consecutivos: RR $<1 \mathrm{~mm}$ & Días & Frecuencia \\
\hline TN90P & $\begin{array}{l}\text { Noches calientes, porcentaje mensual de días en los que la temperatura mínima } \\
\text { supera el percentil 90 TN>TN90P }\end{array}$ & Días & Frecuencia \\
\hline TX90P & $\begin{array}{l}\text { Días calientes, porcentaje mensual de días en los que la temperatura máxima } \\
\text { supera el percentil 90 TX>TX90P }\end{array}$ & Días & Frecuencia \\
\hline DTR & Media Mensual del rango de temperatura diario & ${ }^{\circ} \mathrm{C}$ & \multicolumn{2}{c|}{ Magnitud } \\
\hline TN & Media mensual de la temperatura mínima diaria & ${ }^{\circ} \mathrm{C}$ & ${ }^{\circ}$ Magnitud \\
\hline TX & Media mensual de la temperatura máxima diaria & ${ }^{\circ} \mathrm{C}$ & Magnitud \\
\hline
\end{tabular}


Estimación de la tendencia. El análisis de tendencia, se realizó a dos niveles, regional y local. En el análisis regional, observó las tendencias por década, se empleó una estación para temperatura con datos entre 1976 - 2013 y otra estación para precipitación, con datos entre 1965 - 2009; estas son las únicas estaciones con datos de largo plazo, en el área de estudio. El análisis local observó la variación espacial de las tendencias en el área de estudio y empleó 22 estaciones, con datos de precipitación y de temperatura, entre 1998 2013.

Para el análisis de tendencia de cada uno de los índices de eventos extremos, se realizó la descomposición de la serie en tendencia y en estacionalidad, a través de la función stl en el software R. Este proceso asume que los datos se fraccionan en las diferentes tendencias temporales, empleando procesos de suavización y de regresión local ponderada (Cleveland et al. 1990).

Por otro lado, se utilizó un método robusto no paramétrico, dado que el supuesto de normalidad, en este tipo de datos autocorrelacionados temporalmente, no se cumple. Se estimó la pendiente propuesta por Sen (1968), que es un estimador insesgado de la pendiente en una regresión lineal, cuenta con una alta eficiencia asintótica y es menos sensible a valores extremos; se llevó a cabo en el software $R$, utilizando el paquete mblm. En el análisis, en el nivel local, para cada índice, se elaboró mediante ArcGis, un mapa temático en el que se representaron las estaciones con tendencia positiva o negativa y sin tendencia, así como la significancia un nivel de 0,1 .

\section{RESULTADOS Y DISCUSIÓN}

Tendencias regionales. En el valle geográfico del río Cauca, los índices de eventos extremos de precipitación muestran una tendencia creciente de la lluvia, tanto en el número de días lluviosos como en la cantidad de precipitación. Los días lluviosos (CWD) y sin lluvias (CDD) consecutivos no presentaron tendencia; el índice del total de días húmedos en el mes (TWD) presentó una tendencia por década positiva $(0,492)$ y significativa, lo que se confirma con la tendencia negativa $(-0,456)$, del total de días secos en el mes (TDD); los índices que brindan información sobre la magnitud de la precipitación parecen estar aumentando para la precipitación mensual máxima, en 5 días consecutivos (RX5DAY-0,612 mm), como para la precipitación mensual total (PRCTOT-1,068 $\mathrm{mm}$ ), a pesar que dicha tendencia no es significativa (Tabla 2).

Tabla 2. Tendencia mensual en (días/década) de indicadores de eventos extremos de temperatura entre 1975 - 2013 y precipitación entre 1975 - 2009 y número de estaciones en las que la tendencia entre 1998 y 2013 es positiva y significativa, negativa y significativa y no significativa.

\begin{tabular}{|c|c|c|c|c|c|}
\hline \multicolumn{2}{|c|}{$\begin{array}{c}\text { Índices de extremos } \\
\text { climáticos }\end{array}$} & \multirow{2}{*}{$\begin{array}{c}\begin{array}{c}\text { Nivel Re- } \\
\text { gional }\end{array} \\
\begin{array}{c}\text { Tendencia } \\
\text { década }\end{array}\end{array}$} & \multicolumn{3}{|c|}{ Nivel Local } \\
\hline Nombre & Unidades & & $\begin{array}{c}\text { \# estaciones con } \\
\text { tendencia negativa } \\
\text { (\# estaciones Sig. Al } 0.1 \text { ) }\end{array}$ & $\begin{array}{c}\text { \# estaciones con } \\
\text { tendencia positiva } \\
\text { (\# estaciones Sig. Al } 0.1 \text { ) }\end{array}$ & $\begin{array}{c}\text { \# estaciones sin } \\
\text { tendencia }\end{array}$ \\
\hline \multicolumn{6}{|l|}{ Precipitación } \\
\hline CWD & Días & - & 0 & $1(1)$ & 21 \\
\hline CDD & Días & - & $1(0)$ & 0 & 21 \\
\hline TDD & Días & $-0,456 *$ & $12(0)$ & 0 & 10 \\
\hline RX5DAY & $\mathrm{mm}$ & 0,612 & 0 & $20(2)$ & 2 \\
\hline PRCTOT & $\mathrm{mm}$ & 1,068 & 0 & $22(2)$ & 0 \\
\hline $\mathrm{TX}$ & ${ }^{\circ} \mathrm{C}$ & $0,132 *$ & $10(0)$ & 0 & 12 \\
\hline TN & ${ }^{\circ} \mathrm{C}$ & $0,012 *$ & 0 & $22(13)$ & 0 \\
\hline DTR & ${ }^{\circ} \mathrm{C}$ & $0,007^{*}$ & 0 & $22(16)$ & 0 \\
\hline
\end{tabular}

* Significancia : 0,05. 
Los índices de eventos extremos, basados en la temperatura máxima diaria (TX90P-días calientes, TX-temperatura máxima promedio mensual), presentan tendencia al aumento $(0,780$ y 0,132 , respectivamente), mientras que los basados en la temperatura mínima diaria, como noches cálidas (TN90P), no presenta tendencia y temperatura mínima promedio mensual (TN), muestra una tendencia de 0,012. Por su parte, el rango de la temperatura diurna mensual (DTR) presenta una tendencia significativa de 0,007 (Tabla 2).

Análisis local de índices de extremos de temperatura y precipitación. Todos los índices de precipitación mostraron tendencias positivas o negativas para la precipitación; sin embargo, solo presentaron estaciones significativas los días lluviosos (CWD), la precipitación mensual máxima en 5 días consecutivos (RX5DAY) y la precipitación total (PRCTOT) (Tabla 2).

Los índices de temperatura que representan tendencias al aumento, como el porcentaje mensual de días en los que la temperatura máxima diaria (TX90P) supera el percentil 90, presentan 15 estaciones con tendencia positiva y todas son significativas; las 22 estaciones muestran tendencias positivas para la temperatura máxima y mínima mensual promedio (TX y TN, respectivamente), pero tan solo 13 son significativas. Para la temperatura mínima que supera el percentil 90 (TN90P) fueron 10 estaciones, con tendencia negativa, pero ninguna significativa y el rango (DTR), las 22 estaciones tienen tendencia positiva, con 16 significativas (Tabla 2).

En la figura 1, se presentan las tendencias locales para el CWD, que solo indicó tendencia positiva y significativa al norte del área del estudio; por su parte el TWD presentó algunas estaciones con tendencias positivas, pero no significativas, especialmente, al sur occidente del VGRC. Similarmente, sucede con el CDD y TDD, los cuales, presentaron estaciones con tendencias negativas, pero no significativas. Para el RX5DAY y el PRCTOT, la mayoría de las estaciones muestran tendencia positiva, ubicadas al sur del área de estudio. La dificultad en la identificación de tendencias debe ser abordada en estudios futuros con series, que incluyan un mayor número de años.

Similarmente, en la figura 2, se muestran las tendencias locales para el TX90P, en los que la mayoría de las estaciones ubicadas en el sur y centro del VGRC presentaron tendencias positivas y significativas, lo que sucedió de igual manera con el TX. Por su parte, el TN y el DTR reflejaron tendencia positiva y significativa en más del $50 \%$ de las estaciones. De forma contraria, el TN90P mostró tendencias negativas en algunas de las estaciones, pero no significativas. Cabe destacar, que la ausencia de significancia estadística alta no sugiere que la tendencia no es válida; la significancia es una manera de dar respaldo de manera estadística a los resultados; sin em- bargo, esta dependerá de la longitud de los registros y del porcentaje de datos faltantes.

En Colombia y en Suramérica, se han realizado pocos estudios sobre índices de extremos climáticos, a escala mensual; tradicionalmente, se realizan a escala anual o trimestral, utilizando diferentes métodos, como el RClimDex, Stardex y Mann-Kendall, para encontrar tendencias que contribuyan a la detección de la variabilidad climática (VC) y el cambio climático.

Los índices de eventos climáticos extremos estimados, a escala mensual, pueden bridar una mayor precisión al momento de estimar correlaciones entre clima, dengue y EDAs, debido a que los ciclos de transmisión de estos eventos en salud ocurren en periodos cortos, según la cadena epidemiológica (Organización Panamericana de la Salud, 2002).

Skansi et al. (2013), IDEAM et al. (2017) y Rusticucci (2012) analizaron índices de eventos extremos de temperatura, a escala trimestral, en Cundinamarca, Colombia y anual, en toda Suramérica y en Argentina, respectivamente, y encontraron tendencias al incremento para TX, TN y los días calientes (Tx90p), lo que se presenta, de la misma manera en el presente estudio; sin embargo, se presentan diferencias para las noches calientes (TN90p), pues los resultados obtenidos muestran tendencias negativas, mientras que en Skansi et al. (2013) y Rusticucci (2012), estas son positivas.

Con respecto a los índices asociados a la precipitación, para la precipitación total anual (PRCPTOT), los resultados muestran tendencias al incremento, lo que coincide con resultados obtenidos por Martín-Puertas et al. (2011); sin embargo, estudios realizados, a nivel anual, por Mayorga et al. (2011) encontraron una disminución en las tendencias en el sur occidente colombiano para la precipitación total anual, lo que podría indicar que en algunos meses del año las lluvias son más intensas sin ser tan frecuentes, es decir, hay ocurrencia de eventos extremos máximos de precipitación.

En el caso de los días lluviosos consecutivos, muestra tendencias al incremento; estos resultados coinciden con IDEAM et al. (2017), aunque las tendencias se calcularon a escala trimestral. Para la cantidad de lluvia acumulada en 5 días consecutivos (Rx5day), las tendencias son positivas y coinciden con estudios realizados para Suramérica, por Skansi et al. (2013).

Los eventos climáticos extremos son un factor de riesgo para las enfermedades infecciosas, debido a que los cambios en la temperatura, en la humedad y en el patrón de precipitación, pueden afectar la reproducción, la maduración y la supervivencia de diferentes patógenos (protozoos, bacterias, hongos virus) y vectores, incluido los mosquitos, así como 

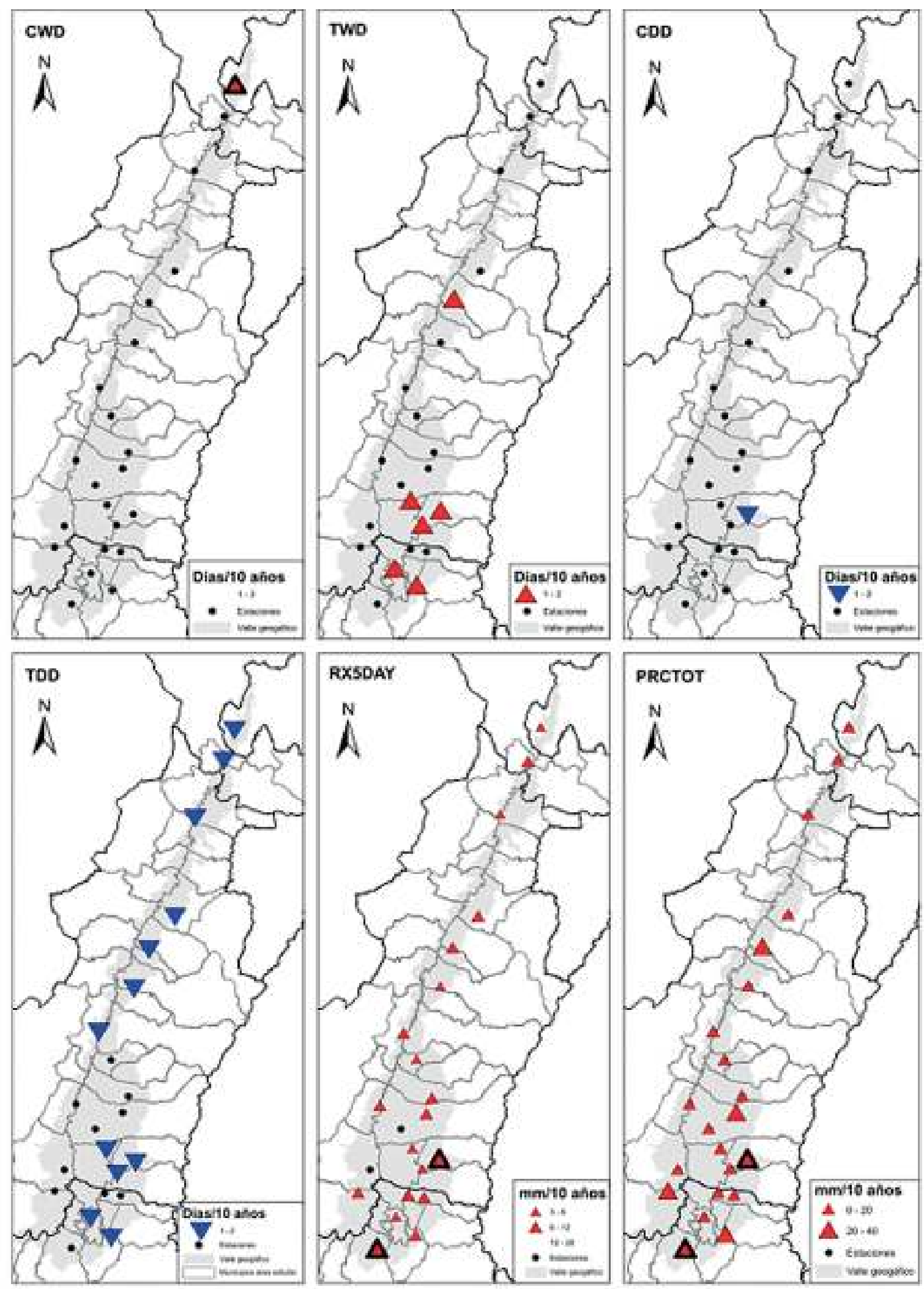

Figura 1. Tendencia local estimada mensualmente para el periodo de 1998 - 2013, para los indicadores de eventos climáticos extremos de precipitación. Los triángulos remarcados son estaciones con tendencias significativas al 0,1. 

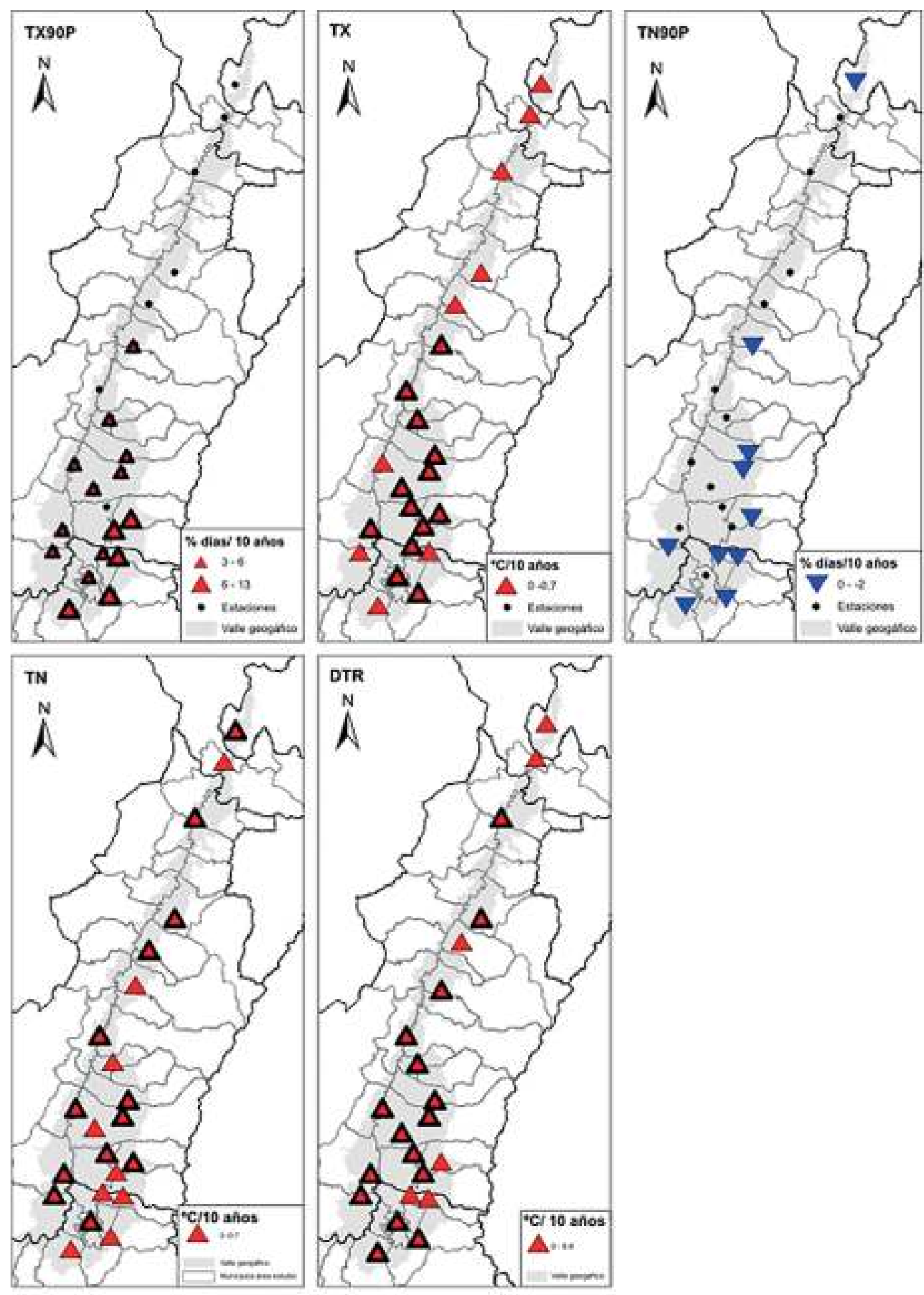

Figura 2. Tendencia local estimada mensualmente para el periodo de 1998 - 2013, para los índices de eventos climáticos extremos de temperatura. Los triángulos remarcados son estaciones con tendencias significativas al 0,1. 
la capacidad para infectar a los humanos, resultando en un incremento de enfermedades infecciosas, como el dengue y las EDA (Patz et al. 2008) (Tabla 3).
El dengue es una de las prioridades en salud pública mundial, pues se estima que para el 2050, 2.800 millones de habitantes estarán en riesgo de sufrirla (Astrom et al. 2012), la cual,

Tabla 3. Efectos posibles en dengue y EDAs, según la tendencia de los eventos climáticos extremos, en el valle geográfico del río Cauca.

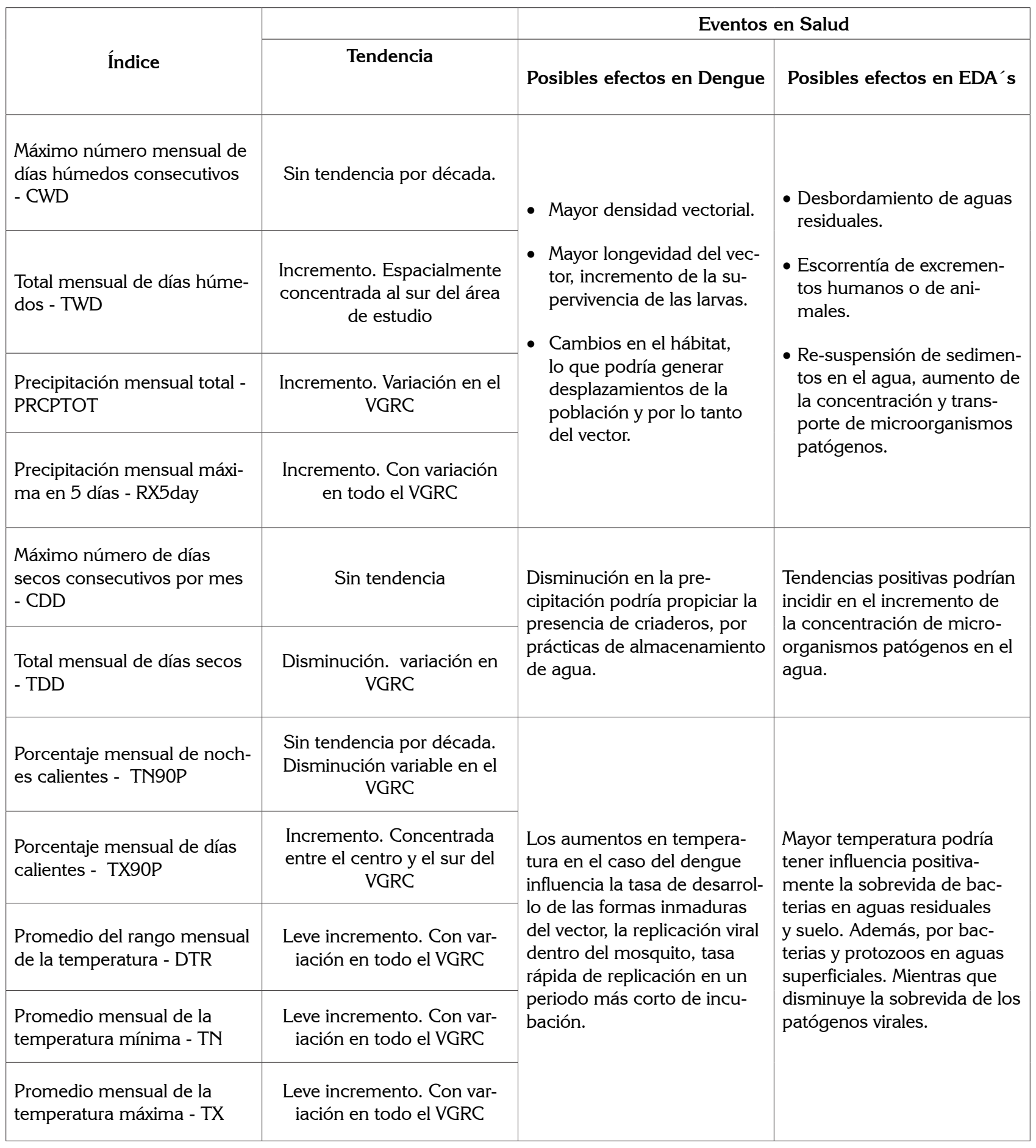


se puede incrementar, dado a que las áreas que climáticamente favorecen la ocurrencia de dengue están en aumento (IPCC, 2014). Adicionalmente, en países como México, se proyecta que la incidencia aumentará un $40 \%$, en el 2080 , a consecuencia del cambio climático (Colón-González et al. 2013). Por su parte, la EDA es la segunda causa de muerte de menores de 5 años y se estimó para el siglo XX que el cambio climático fue la causa de un aumento de $2,4 \%$, en los casos de diarrea, pero además en el 2011, se proyectó un incremento entre 8-11\%, en el riesgo de diarrea en áreas tropicales y subtropicales, en el 2039, a consecuencia del cambio climático, usando un escenario A1B (IPCC, 2014).

En cuanto a eventos extremos de precipitación, Chen et al. (2012), en Taiwan, encontraron que la incidencia de dengue se ve afectada por niveles de lluvia, hasta de $350 \mathrm{~mm} /$ día; sin embargo, esta es más alta en eventos de lluvia $<130 \mathrm{~mm} /$ día, mientras que Colón-González et al. (2013), en México, encontraron un aumento de la incidencia de la enfermedad, con precipitaciones promedio mensuales, que oscilan entre 500-600mm. En relación a la temperatura, Wu et al. (2009) detectaron en Taiwan, como asociaciones más importantes: máxima temperatura mensual $(R=0,24, p<0,05)$, temperatura mínima mensual $(R=0,23, p<0,5)$, humedad relativa $(R=0,2, p<0,05)$, con rezagos de dos meses. Además, hallaron correlaciones significativas entre la precipitación y la incidencia de dengue $(R=0,37, p<0,05)$.

Los hallazgos del presente estudio muestran una tendencia hacia el aumento del número de días lluviosos y de la cantidad de precipitación. La precipitación tiene el potencial de incrementar la densidad del vector adulto y tendría efecto en la longevidad, al aumentar la supervivencia de las larvas. La humedad generaría un hábitat favorable para el desarrollo del mosquito y aumentaría su sobrevida. Por otro lado, las inundaciones ocasionarían cambios en el hábitat, lo que podría generar desplazamientos de la población y, por lo tanto, del vector. Estos fenómenos también ocasionarían la implementación de mayores prácticas de almacenamiento de agua en la población, por disminución de disponibilidad (Halstead, 2008; Morin et al. 2013; Naish et al. 2014).

Los índices de eventos extremos de temperatura (TX90P_ días calientes, TX-Temperatura máxima promedio mensual) presentan tendencia al aumento y pueden generar oleadas de calor, escasez de agua, alta evapotranspiración, entre otras. Por otro lado, favorecen las condiciones para el ciclo de vida de vectores, como el del dengue, además de su presencia en zonas donde históricamente no existían; además, acortan la duración del ciclo de vida del mosquito, que es el tiempo en que ocurre la propagación sistémica del virus, dentro del mosquito. A $32^{\circ} \mathrm{C}$, los mosquitos pueden tener 2,6 veces la oportunidad de completar un periodo extrínse- co, en comparación con temperaturas de $24^{\circ} \mathrm{C}$ (Halstead, 2008; Morin et al. 2013; Naish et al. 2014).

Con respecto a las EDA, Moors et al. (2013) evaluaron el efecto de cambio climático sobre la incidencia de diarrea en la India, encontrando que el incremento en la temperatura aumentaba la ocurrencia de EDA en $10 \%$ y la humedad relativa lo hacía entre el 2 y $4 \%$ y al disminuir la precipitación, la incidencia aumenta entre 2 y $8 \%$. De igual forma, Bandyopadhyay et al. (2012) muestran que los aumentos en la temperatura máxima aumentan también la prevalencia de EDA, en África Sub-sahariana. En Taiwan, se encontró que un incremento en la precipitación superior a 350mm/día aumentaba la incidencia de diarrea, por disentería bacilar y por virus (Chen et al. 2012).

En este sentido, la tendencia de precipitación encontrada podría aumentar el riesgo de desbordamientos de aguas residuales, de escorrentías, de excrementos humanos o de animales, de re-suspensión de sedimentos, lo que aumentaría la concentración y el transporte de microorganismos patógenos y contaminación de las aguas superficiales y subterráneas (Moors et al. 2013), lo que lleva al aumento de los niveles de indicadores bacterianos y patógenos, en potenciales aguas de consumo humano. Asimismo, las inundaciones podrían contaminar las aguas subterráneas, por infiltración y por flujo entre las tuberías de alcantarillado y de agua potable.

Las áreas que podrían tener una mayor afectación, se encuentran ubicadas al sur del valle geográfico del río Cauca, que presentan, al mismo tiempo, tendencias crecientes para eventos climáticos extremos de precipitación y de temperatura. Los municipios localizados en esta área deben desarrollar estrategias orientadas a valorar la vulnerabilidad, para desarrollar estrategias de adaptación en salud (Organización Panamericana de la Salud, 2011).

La principal fortaleza de contar con datos de eventos climáticos extremos calculados a nivel mensual es el potencial que estos tienen por encima de los calculados, a nivel anual, para evaluar la relación con eventos en salud, debido a que el proceso de la evolución del dengue y las EDA se presenta a escalas cortas, como las mensuales; sin embargo, aunque los índices calculados, a escala anual, no brindan una relación directa con los eventos de salud, si son una buena base para el estudio de los efectos sobre la salud humana. Es el caso de la variabilidad interanual del fenómeno ENOS, en donde se siguen tendencias en las series históricas, para detectar cambios entre la ocurrencia de eventos en salud y su relación con el clima y el cambio climático.

Futuros estudios deberían analizar correlaciones de series de tiempo de los eventos extremos y de las series de casos de 
las enfermedades asociadas a los mismos, así como estudios que relacionan el ENOS con brotes epidémicos (Brunkard et al. 2008; Siraj et al. 2014). De igual manera, se requieren estudios interdisciplinarios de la relación clima - salud, que aborden las amenazas emergentes relacionadas con la VC y el cambio climático, así como la relación con nuevos virus, como el Zika y el Chikunguña y los impactos de los mismos.

Agradecimientos: Al International Development Research Centre - IDRC, a los grupos GESP e IREHISA de la Universidad del Valle, al Programa de Jóvenes Investigadores e Innovadores y programa de generación bicentenario de formación doctoral de COLCIENCIAS; Corporación Regional del Valle del Cauca (CVC), IDEAM y CENICAÑA, por el suministro de la información. Conflicto de intereses: El manuscrito fue preparado y revisado con la participación de todos los autores, quienes declaramos que no existe conflicto. Financiación: Este estudio fue financiado por International Development Research Centre - IDRC.

\section{BIBLIOGRAFÍA}

1. AGUILAR, E.; AUER, I.; BRUNET, M.; PETERSON, T.C.; WIERINGA, J. 2003. Guidance on metadata and homogenization. Switzerland: World Meteorological Organization. 1186:53.

2. ASTROM, C.; ROCKLOV, J.; HALES, S.; BEGUIN, A.; LOUIS, V.; SAUERBORN, R. 2012. Potential distribution of dengue fever under scenarios of climate change and economic development. Ecohealth. (EEUU). 9(4):448-454.

3. BANDYOPADHYAY, S.; KANJI, S.; WANG, L. 2012. The impact of rainfall and temperature variation on diarrheal prevalence in Sub-Saharan Africa. Applied Geography. (EEUU). 33:63-72.

4. BRUNKARD, J.M.; CIFUENTES, E.; ROTHENBERG, S.J. 2008. Assessing the roles of temperature, precipitation, and ENSO in dengue re-emergence on the Texas-Mexico border region. Salud Pública Mex. 50(3):227-234.

5. CAI, W.; WANG, G.; SANTOSO, A.; MCPHADEN, M.J.; WU, L.; JIN, F.; TIMMERMANN, A.; COLLINS, M.; VECCHI, G.; LENGAIGNE, M.; ENGLAND, M.H.; DOMMENGET, D.; TAKAHASHI, K.; GUILYARDI, E. 2015. Increased frequency of extreme La Nina events under greenhouse warming. Nature Clim. Change. (EEUU). 5(2):132-137.

6. CLEVELAND, R.B.; CLEVELAND, W.S.; MCRAE, J.E.; TERPENING, I. 1990. STL: A seasonal-trend decom- position procedure based on loess. Officlal Statistics. (Poland). 6(1):3-33.

7. COLÓN-GONZÁLEZ, F.J.; FEZZI, C.; LAKE, I.R.; HUNTER, P.R. 2013.The effects of weather and climate change on dengue. PLoS Neglected Tropical Diseases. (EEUU).7(11):e2503.

8. CHEN, M.-J.; LIN, C.-Y.; WU, Y.-T.; WU, P.-C.; LUNG, S.C.; SU, H.-J.; 2012. Effects of extreme precipitation to the distribution of infectious diseases in Taiwan, 1994-2008. PLoS ONE. (EEUU).7(6):e34651.

9. FORERO, E.L.; HERNÁNDEZ, Y.T.; ZAFRA, C.A. 2014. Percepción latinoamericana de cambio climático: metodologías, herramientas y estrategias de adaptación en comunidades locales. Una revisión. Rev. U.D.C.A Act. \& Div. Cient. (Colombia). 17:73-85.

10. FRIEDRICH, M.J. 2013. Climate change linked with increase in diarrheal disease. JAMA. (EEUU). 309(19):1985-1985.

11. HALSTEAD, S.B. 2008. Dengue virus-mosquito interactions. Ann. Rev Entomol. (EEUU). 53:273-291.

12. HOYOS, I.; BAQUERO, A.; JACOB, D.; RODRIGUEZ, B. 2012. Variability of extreme events in the Colombian pacific and Caribbean catchment basins. Clim Dyn. DOI 10.1007/s00382-012-1487-9.

13. IDEAM; PNUD; MADS; DNP; CANCILLERÍA. 2017. Tercera Comunicación Nacional De Colombia a La Convención Marco De Las Naciones Unidas Sobre Cambio Climático (CMNUCC). Tercera Comunicación Nacional de Cambio Climático. IDEAM, PNUD, MADS, DNP, CANCILLERÍA, FMAM. Bogotá D.C., Colombia.

14. INTERGOVERNMENTAL PANEL ON CLIMATE CHANGE -IPCC-. 2012. Gestión de los riesgos de fenómenos meteorológicos extremos y desastres para mejorar la adaptación al cambio climático. p.1-32.

15. INTERGOVERNMENTAL PANEL ON CLIMATE CHANGE IPCC-. 2014. Human Health: impacts, adaptation and Co-benefits. In contribution Wgl, editor. Climate change 2014: Impacts, adaptation, and vulnerability Part A: Global and sectoral aspects. (EEUU). p.709-754.

16. KOVATS, R.S.; CAMPBELL-LENDRUM, A.; MATTHIES, F. 2005. Climate change and human health: estimating avoidable deaths and disease. Risk analysis. (EEUU). 25(6):1409-1418. 
17. MARTÍN-PUERTAS, C.; DORADO-LINAN, I.; BRAUER, A.; ZORITA E.; VALERO-GARCÉS, B.L.; GUTIERREZ, E. 2011. Hydrological evidence for a North Atlantic oscillation during the Little Ice Age outside its range observed since 1850. Clim. Past Discuss. 7:4149-4171.

18. M.G., A.; TANK, K; ZWIERS, F.W.; ZHANG. X. 2009. Guidelines on Analysis of extremes in a changing climate in support of informed decisions for adaptation. World Meteorological Organization. (Switzerland).

19. MAYORGA, R.; HURTADO, G.; BENAVIDES, H. 2011. Evidencias de cambio climático en Colombia con base en información estadística. Instituto de Hidrología, Meteorología y Estudios Ambientales-IDEAM. (Colombia).

20. MCMICHAEL, A.J.; WOODRUFF, R.E. 2005. Climate change and human health. Encyclopedia of World Climatology. Springer Netherlands.

21. MONTEALEGRE, E. 2012. Análisis de la variabilidad climática inter-anual (El Niño y La Niña) en la Región Capital, Bogotá Cundinamarca Plan Regional Integral de Cambio Climático Región Capital Bogotá Cundinamarca (PRICC). p.1-73.

22. MOORS, E.; SINGH, T.; SIDERIUS, C.; BALAKRISHNAN, S.; MISHRA, A.; 2013. Climate change and waterborne diarrhoea in northern India: Impacts and adaptation strategies. Science of the Total Environment. (Netherlands). 468:S139-S51.

23. MORIN, C.W.; COMRIE, A.C.; ERNST, K. 2013. Climate and dengue transmission: evidence and implications. Environ. Health Perspect. (EEUU). 121(11-12):12641272.

24. NAISH, S.; DALE, P.; MACKENZIE, J.S.; MCBRIDE, J.; MENGERSEN, K.; TONG, S. 2014. Climate change and dengue: a critical and systematic review of quantitative modelling approaches. BMC infectious diseases. (United Kingdom). 14(1):167-181.

25. NATIONAL CLIMATIC DATA CENTER -NCDC-. 1997. 1961 - 1990 WMO global standar Climatic Normals. Disponible desde internet en: http://www.ncdc.noaa. gov/oa/climate/research/normals/wmo/wmo-normals.html (con acceso 21/07/2014).

26. ORGANIZACIÓN PANAMERICANA DE LA SALUD. 2002. Módulos de Principios de Epidemiología para el Con- trol de Enfermedades, segunda edición. Washington D.C.: OPS. p.1-46.

27. ORGANIZACIÓN PANAMERICANA DE LA SALUD. 2011. Lineamientos para evaluar la vulnerabilidad de la salud frente al cambio climático en Colombia: Documento de apoyo para las autoridades de salud en Colombia. Bogotá. p.1-56.

28. PATZ, J.A.; VAVRUS, S.J.; UEJIO, C.K.; MCLELLAN, S.L. 2008. Climate change and waterborne disease risk in the Great Lakes Region of the U.S. Am. J. Preventive Medicine. (EEUU). 35(5):451-458.

29. PETERSON, T.C.; EASTERLING, D.R.; KARL, T.R.; GROISMAN, P.; NICHOLLS, N.; PLUMMER, N.; VINCENT, L. 1998. Homogeneity adjustments of in situ atmospheric climate data: a review. Int. J. Climatology. (EEUU). 18(13):1493-1517.

30. POVEDA, G.; ÁLVAREZ, D.; RUEDA, Ó. 2011. Hydroclimatic variability over the Andes of Colombia associated with ENSO: a review of climatic processes and their impact on one of the Earth's most important biodiversity hotspots. Clim. Dyn. (EEUU). 36(1112):2233-2249.

31. REBOITA, M.S; KRUSCHE, N.; AMBRIZZI, T.; DA ROCHA, R.P. 2012. Entendendo o Tempo e o Clima na América do Sul. Terræ didatica 8(1):34-50.

32. RUSTICUCCI, M. 2012. Observed and simulated variability of extreme temperature events over South America. Atmospheric Res. (EEUU). 106:1-17.

33. SEN, P.K. 1968. Estimates of the regression coefficient based on Kendall's Tau. J. Am. Statistical Assoc. (EEUU). 63(324):1379-1389.

34. SIRAJ, A.S.; SANTOS-VEGA, M.; BOUMA, M.J.; YADETA, D.; CARRASCAL, D.R.; PASCUAL, M. 2014. Altitudinal changes in malaria incidence in highlands of Ethiopia and Colombia. Science. (EEUU).343(6175):1154-1158.

35. SKANSI, M.M.; BRUNET, M.; SIGRÓ, J.; AGUILAR, E.; AREVALO-GROENING, J.A.; BENTANCUR, O.J.; CASTELLÓN, Y.R.; CORREA, R.L.; JÁCOME, H.; MALHEIROS, A.; ORIA, C.; MAX, A.; SALLONS, S.; VILLAROEL, C.; MARTINEZ, R.; ALEXANDER, L. 2013. Warming and wetting signals emerging from analysis of changes in climate extreme indices over South America. Global and Planetary Change. (EEUU). 100:295-307. 
36. VELANDIA, M.L.; CASTELLANOS, J.E. 2011 Virus del Recibido: Agosto 24 de 2016 dengue: estructura y ciclo viral. Infectio. (Colombia). Aceptado: Octubre 26 de 2017 15:33-43.

37. WU, P.C.; LAY, J.G.; GUO, H.R.; LIN, C.Y.; LUNG, S.C.; SU, H.J. 2009. Higher temperature and urbanization affect the spatial patterns of dengue fever transmission in subtropical Taiwan. Science of the Total Environment. 407(7):2224-2233.

Cómo citar:

Cuartas, D.E.; Caicedo, D.M.; Ortega, D.; Cardona, F.; Carvajal, Y.; Méndez, F. 2017. Tendencia espacial y temporal de eventos climáticos extremos en el valle geográfico del Río Cauca. Rev. U.D.C.A Act.\& Div. Cient. 20(2): 267-278. 